\title{
Lost and Forgotten? Orientation Versus Memory in Alzheimer's Disease and Frontotemporal Dementia
}

\author{
Belinda Yew $^{\mathrm{a}}$, Suvarna Alladi ${ }^{\mathrm{d}}$ Mekala Shailaja $^{\mathrm{d}}$, John R. Hodges ${ }^{\mathrm{a}, \mathrm{b}, \mathrm{c}}$ and Michael Hornberger ${ }^{\mathrm{a}, \mathrm{b}, \mathrm{c}, *}$ \\ ${ }^{a}$ Neuroscience Research Australia, Sydney, Australia \\ ${ }^{\mathrm{b}}$ Faculty of Medicine, University of New South Wales, Sydney, NSW, Australia \\ ${ }^{\mathrm{c}}$ ARC Centre of Excellence in Cognition and its Disorders, Sydney, NSW, Australia \\ ${ }^{\mathrm{d}}$ Department of Neurology, Nizam's Institute of Medical Sciences, Hyderabad, India
}

Handling Associate Editor: Paulo Caramelli

Accepted 9 August 2012

\begin{abstract}
Recent studies suggest that significant memory problems are not specific to Alzheimer's disease (AD) but can be also observed in other neurodegenerative conditions, such as behavioral variant frontotemporal dementia (bvFTD). We investigated whether orientation (spatial \& temporal) information is a better diagnostic marker for AD compared to memory and whether their atrophy correlates of orientation and memory differ. A large sample $(n=190)$ of AD patients $(n=73)$, bvFTD patients $(n=54)$, and healthy controls $(n=63)$ underwent testing. A subset of the patients $(n=72)$ underwent structural imaging using voxel-based morphometry analysis of magnetic resonance brain imaging. Orientation and memory scores from the Addenbrooke's Cognitive Examination showed that AD patients had impaired orientation and memory, while bvFTD patients performing at control level for orientation but had impaired memory. A logistic regression showed that $78 \%$ of patients could be classified on the basis of orientation and memory scores alone at clinic presentation. Voxel-based morphometry analysis was conducted using orientation and memory scores as covariates, which showed that the neural correlates for orientation and memory also dissociated with posterior hippocampus cortex being related to orientation in $\mathrm{AD}$, while the anterior hippocampus was associated with memory performance in the AD and bvFTD patients. Orientation and memory measures discriminate AD and bvFTD to a high degree and tap into different hippocampal regions. Disorientation and posterior hippocampus appears therefore specific to AD and will allow clinicians to discriminate $\mathrm{AD}$ patients from other neurodegenerative conditions with similar memory deficits at clinic presentation.
\end{abstract}

Keywords: Alzheimer's disease, atrophy, frontotemporal dementia, hippocampus, memory, orientation

Supplementary data available online: http://dx.doi.org/10.3233/JAD-2012-120769

\section{INTRODUCTION}

One of the clinical features traditionally regarded as differentiating Alzheimer's disease (AD) from other

${ }^{*}$ Correspondence to: Dr. Michael Hornberger, Neuroscience Research Australia, Sydney, Randwick, NSW 2031, Australia. Tel.: +61 29399 1816; E-mail: m.hornberger@neura.edu.au. dementias, including frontotemporal dementia (FTD), is performance on memory tests. Indeed, for some forms of frontotemporal dementia, such as behavioral variant frontotemporal dementia (bvFTD), substantial episodic memory impairment is regarded as a diagnostic exclusion criterion [1]. Recent evidence [2], however, suggests that a large percentage of bvFTD patients present with episodic memory problems. 
Single case reports [3] have confirmed that patients with pathologically confirmed bvFTD can have severe amnesia, while a retrospective study [2] of bvFTD cases, a proportion of whom came to autopsy, showed that a high percentage had memory impairment of the same magnitude as seen in AD. This is further corroborated via imaging studies showing similar levels of hippocampal atrophy in bvFTD and AD [4-6], although this can vary for bvFTD patients [7]. A potential diagnostic distinction between $\mathrm{AD}$ and bvFTD, however, may be the ability to correctly identify one's location in space and time. AD patients are known to experience disorientation from an early stage [8]; clinical reports suggest that orientation is intact in bvFTD cases [2], although this has not been explored systematically.

We investigated memory and orientation in a large sample of $\mathrm{AD}$ and bvFTD patients. We predicted memory deficits in both groups, while only the AD patients should show disorientation. In a second step, we compared performance on more specific memory (i.e., immediate versus delayed anterograde recall) and orientation (i.e., temporal versus spatial orientation) measures to explore their diagnostic utility. Finally, we employed voxel-based morphometry to identify the grey matter atrophy regions that covary with the orientation and memory performance in the patients.

\section{MATERIALS AND METHODS}

\section{Case selection}

A sample of $73 \mathrm{AD}$ and 54 bvFTD consecutively seen patients were sourced from the FRONTIER Dementia Clinic database, Sydney, Australia and the Memory Clinic, Hyderabad, India. All FTD patients met current consensus FTD criteria [1] and showed disease progression as well as atrophy on scans to exclude any phenocopy FTD cases. AD patients fulfilled NINCDS-ADRDA criteria for probable AD [9]. Sixty-three healthy controls (volunteer panel recruits or spouses/carers of patients) matched for gender and education to the AD patients served as controls. The study was approved by the University of New South Wales Human Research Ethics Advisory panel D (Biomedical, ref. \#10035). Clinical, neuropsychological, and demographics data (Table 1) were available for all participants. High-resolution coronal T1 MR brain images were available for all the Sydney patients (33 AD, 39 bvFTD). All data was collected at the first clinic presentation of the patients.

\section{Test selection}

The Addenbrooke's Cognitive ExaminationRevised (ACE-R) [10] was administered to all participants, which contains temporal $(n=5)$ and geographical $(n=5)$ orientation questions. Clinicians also completed the Clinical Dementia Rating Scale (CDR) [11].

ACE-R scores were taken from response to the temporal and geographical components. Overall orientation was calculated by summing these for a total score out of 10 . Immediate and delayed recall were scored out of 7 based on performance on the ACE$\mathrm{R}$ anterograde (name and address learning) and recall tasks. Total memory (scored out of 19) was calculated by summing scores for both anterograde ACE-R memory components (i.e., immediate and delayed recall) as well as the recognition score (scored out of 5).

\section{Voxel-based morphometry analysis}

3D T1-weighted sequences (coronal orientation, matrix $256 \times 256,200,1 \times 1 \mathrm{~mm}^{2}$ in-plane resolution, slice thickness $1 \mathrm{~mm}, \mathrm{TE} / \mathrm{TI}=2.6 / 5.8 \mathrm{~ms}$ ) were analyzed with FSL-VBM, a voxel-based morphometry analysis [12, 13] which is part of the FSL software package http://www.fmrib.ox.ac.uk/fsl/fslvbm/ index.html [14]. First, tissue segmentation was carried out using FMRIB's Automatic Segmentation Tool (FAST) [15] from brain extracted images. The resulting grey matter partial volume maps were then aligned to the Montreal Neurological Institute standard space (MNI152) using the nonlinear registration approach using FNIRT, which uses a b-spline representation of the registration warp field [16]. The registered partial volume maps were then modulated (to correct for local expansion or contraction) by dividing them by the Jacobian of the warp field. The modulated images were then smoothed with an isotropic Gaussian kernel with a standard deviation of $3 \mathrm{~mm}$ (FWHM: $8 \mathrm{~mm}$ ). A voxelwise general linear model was applied and permutation-based non-parametric testing (5000 permutations) was used to form clusters with the Threshold-Free Cluster Enhancement method [17]. Differences in cortical grey matter intensities between patients (bvFTD and AD) and Controls were assessed using $t$-tests and were tested for significance at $p<0.05$ corrected for family-wise error (FWE) correction (see Supplementary Figure 1). Covariate analyses between memory and orientation scores and regions of grey matter atrophy were investigated in bvFTD and AD patients, separately. For statistical power, 
a covariate only statistical model with a [1] $t$-contrast was used, providing an index of association between decreasing grey matter volume and lower scores on the experimental measures. Importantly, memory and orientation measures were demeaned for the analysis. All clusters in the covariate analyses were tested for significance at $p<0.001$, uncorrected with a voxel threshold of at least 20 contiguous voxels.

\section{Statistics}

Data were analyzed using SPSS 18.0 (SPSS Inc., Chicago, IL). Demographic (age and education) and general cognitive (ACE-R) data were compared across groups via one-way ANOVAs followed by Tukey posthoc tests. For analyses of orientation and memory subscores, CDR was entered as a covariate to control for differences in disease severity. Comparisons across centers (Sydney versus Hyderabad) showed no significant difference for any of the demographic and cognitive (memory, orientation) measures.

\section{RESULTS}

\section{Demographics and global cognitive function}

Comparisons of education between groups yielded no significant differences (Table 1). BvFTD cases were, however, significantly younger than AD patients $(p<0.001)$ and controls $(p<0.001)$. BvFTD and AD patients also differed significantly on CDR score $(p<0.05)$, however were matched for disease duration $(p>0.1)$.

Performance of both patient groups on the overall ACE-R was significantly $(p<0.001)$ worse than that of controls (see Table 1). Post-hoc tests revealed also worse ACE-R performance in AD patients compared to bvFTD patients $(p<0.001)$.

\section{Orientation}

Overall, group comparisons showed significant differences across participants (all $p$ 's $<0.001$ ). Follow-up tests showed that ACE-R orientation scores (temporal, spatial, and overall orientation) were not significantly different for bvFTD and controls (Table $1 \&$ Fig. 1a). By contrast, AD patients were significantly impaired in comparison to controls and bvFTD (Table $1 \&$ Fig. 1a). Pair-wise comparisons of temporal and spatial orientation subtests showed that the overall group performed significantly better for the spatial than the temporal orientation $(p<0.001)$. This effect was driven by AD and bvFTD groups which both showed significantly better spatial than temporal orientation ( $p<0.001$ and $p<0.025$, respectively), whereas the controls did not differ significantly on these measures $(p>0.1)$.

\section{Memory}

Comparisons of ACE-R memory scores showed significant group differences (all $p$ 's $<0.001$ ) (Table 1 \& Fig. 1b) with no significant differences between

Table 1

Demographics \& cognitive tests. Mean (SD) scores and comparisons of patients and controls on demographics and cognitive tests

\begin{tabular}{|c|c|c|c|c|c|c|c|}
\hline & $\mathrm{AD}$ & bvFTD & Controls & $F / K-S$ & $\begin{array}{l}\text { AD versus } \\
\text { controls }\end{array}$ & $\begin{array}{l}\text { bvFTD versus } \\
\text { controls }\end{array}$ & $\begin{array}{l}\text { AD versus } \\
\text { bvFTD }\end{array}$ \\
\hline$n$ & 73 & 54 & 63 & - & - & - & - \\
\hline Age & $65.2(7.5)$ & $59.6(10.4)$ & $65.5(5.9)$ & $* * *$ & n.s. & $* * *$ & $* * *$ \\
\hline Education (Sydney only) & $12.3(3.0)$ & $11.7(2.9)$ & $12.0(2.4)$ & n.s. & - & - & - \\
\hline Gender $(\mathrm{M} / \mathrm{F})$ & $36 / 37$ & $37 / 17$ & $36 / 27$ & - & - & - & - \\
\hline Disease duration (y) & $4.1(3)$ & $3(2.1)$ & - & n.s. & - & - & - \\
\hline ACE-R (100) & $65.0(17.7)$ & $75.6(15.1)$ & $94.0(4.4)$ & $* * *$ & $* * *$ & $* * *$ & $* * *$ \\
\hline CDR & $0.9(0.5)$ & $1.2(0.7)$ & - & $*$ & - & - & $*$ \\
\hline \multicolumn{8}{|l|}{ ACE-R - orientation } \\
\hline Temporal (5) & $2.5(1.7)$ & $3.9(1.3)$ & $4.9(0.3)$ & $* * *$ & $* * *$ & n.s. & $* * *$ \\
\hline Spatial (5) & $3.6(1.4)$ & $4.5(0.8)$ & $5.0(0.2)$ & $* * *$ & $* * *$ & n.s. & $* * *$ \\
\hline Overall (10) & $6.1(2.6)$ & $8.4(1.9)$ & $9.9(0.3)$ & $* * *$ & $* * *$ & n.s. & $* * *$ \\
\hline \multicolumn{8}{|l|}{ ACE-R - memory } \\
\hline Immediate recall (7) & $4.2(2.2)$ & $5.8(1.5)$ & $7.0(0.1)$ & $* * *$ & $* * *$ & n.s. & $* * *$ \\
\hline Delayed recall (7) & $1.6(2.0)$ & $3.0(2.3)$ & $5.9(1.4)$ & $* * *$ & $* * *$ & $* *$ & $* * *$ \\
\hline Recognition (5) & $3.0(1.9)$ & $3.9(1.3)$ & $4.8(0.5)$ & $* * *$ & $* * *$ & n.s. & $* *$ \\
\hline Total (19) & $12.8(4.1)$ & $16.7(1.9)$ & $17.6(1.7)$ & $* * *$ & $* * *$ & n.s. & $* * *$ \\
\hline
\end{tabular}

$F$ values indicate significant differences across groups; Tukey post hoc tests compare differences between group pairs. $* p<0.05 ; * * p<0.01 ; * * * p<0.001$. 
a

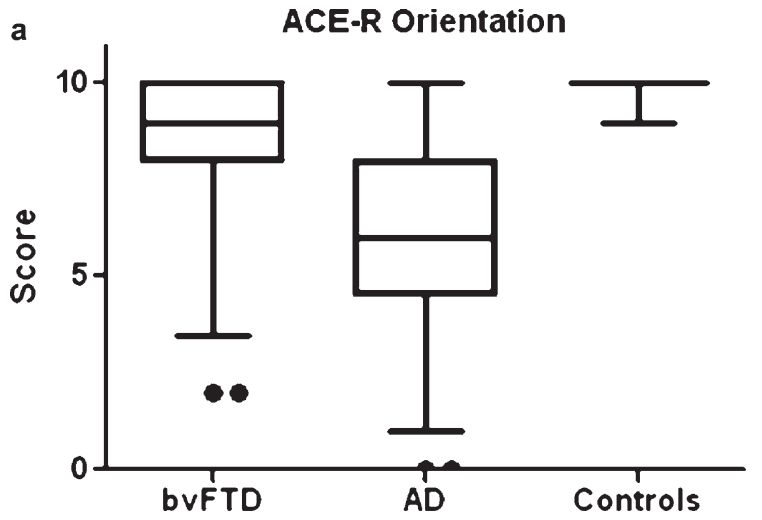

b

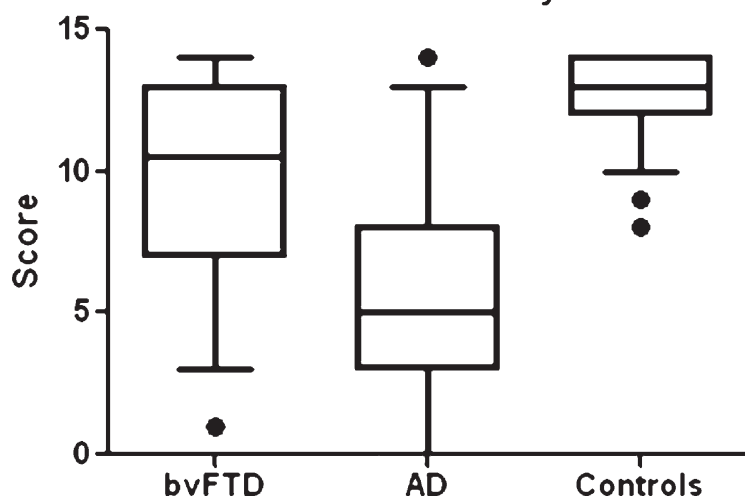

Fig. 1. Orientation and memory scores. Figure 1 shows boxplots of overall orientation (a) and overall memory (b) scores on the Addenbrookes Cognitive examination Revised (ACE-R). Whiskers indicate 5-95 percentile.

bvFTD and controls for ACE-R immediate recall and recognition performance, whereas $\mathrm{AD}$ patients performed significantly worse than bvFTD $(p<0.001)$ and controls $(p<0.001)$. ACE-R delayed recall and total memory differed significantly among groups, with controls outperforming both patient groups (Table 1 $\&$ Fig. 1b) and bvFTD patients performing better than AD patients $(p<0.001)$.

\section{Logistic regression}

We employed the overall orientation and memory scores in a logistic regression employing the Enter method with diagnosis (bvFTD versus AD) as the dependent factor. Controls were excluded from the analysis. Results showed that $78 \%$ of patients (bvFTD: $72.2 \%$; AD: $82.2 \%$ ) could be classified correctly on a combination of overall orientation and memory scores from the ACE-R. Overall orientation alone classified $74 \%$ of patients correctly (bvFTD: $66.7 \%$; AD:
$79.5 \%$ ), whereas overall memory classified only $69 \%$ correctly (bvFTD: 64.8\%; AD: 72.6\%).

\section{Voxel-based morphometry}

As evident in Figs. 2, 3 and Table 2, AD patients' overall orientation performance covaried with posterior hippocampus atrophy $(r=0.557, p<0.01)$. By contrast, ACE-R memory performance correlated with more anterior hippocampus regions in AD (Figs. 2, 3 and Table 2) $(r=0.522, p<0.025)$. Importantly, both spatial and temporal orientation performance also correlated with posterior hippocampus atrophy (Fig. 4) ( $r=0.527, p<0.025 ; r=0.545, p<0.01$; respectively). In addition, the spatial orientation performance also covaried with more anterior hippocampal atrophy, similar to the memory performance region (Fig. 4) $(r=0.565, p<0.01)$. For the bvFTD patients, only the overall memory score significantly correlated with more anterior/middle hippocampus atrophy (Fig. 2) $(r=0.562, p<0.01)$.

\section{DISCUSSION}

In keeping with the starting hypothesis, we found distinct patterns of performance on measures on episodic memory and orientation in bvFTD and AD patients which could help diagnosis of these patient groups. Only those patients with AD showed disorientation. By contrast, memory impairments were evident in both patient groups although the $\mathrm{AD}$ patients showed more severe impairment. Moreover, the neural correlates in $\mathrm{AD}$ showed that the posterior hippocampus covaried with the performance on the orientation scores, while the memory performance was more affected by anterior hippocampal regions. In the bvFTD group, only the memory performance covaried with more anterior hippocampal atrophy.

Behaviorally, bvFTD patients performed at an equivalent level to controls for spatial and temporal orientation. This confirms the clinical impression but has not previously been documented [18]. By contrast, $\mathrm{AD}$ patients were poorly orientated for time and place in keeping with numerous prior observations [8, 18]. Analysis of the memory scores from the ACE-R revealed a different picture, with both patients groups showing impairment in comparison to controls with the exception of immediate recall and learning of the name and address from the ACE-R, which was preserved in bvFTD. Furthermore, bvFTD and AD patients differed on the memory scores with more severe impairment in 

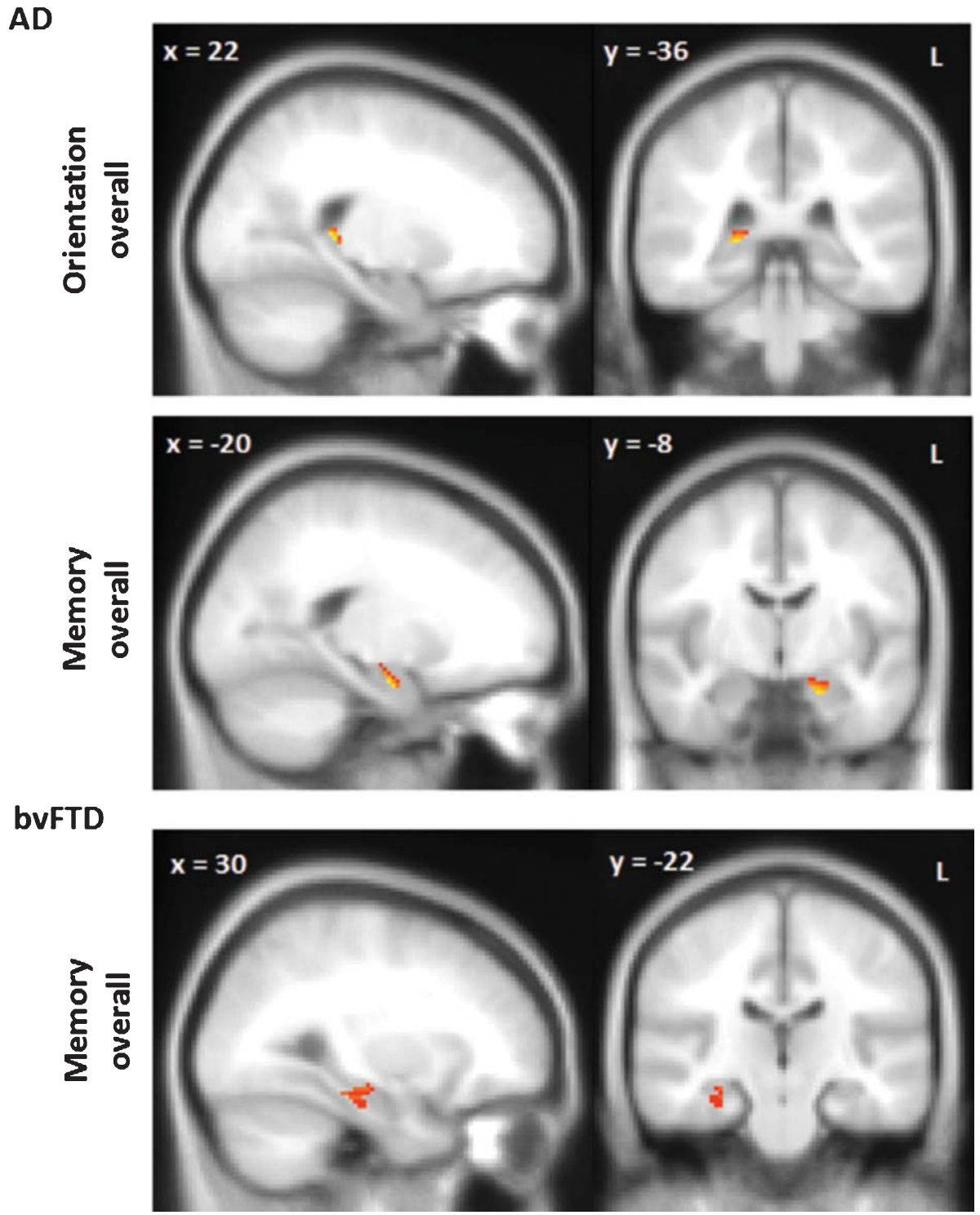

Fig. 2. Brain atrophy related to orientation and memory. Voxel-based morphometry analyses showing grey matter atrophy covarying with overall orientation and memory scores for AD and bvFTD. Clusters are overlaid on the MNI standard brain $(t>2.41)$. Colored voxels show regions that were significant in the analyses for $p<0.0001$ uncorrected and a cluster threshold of 20 contiguous voxels.

AD. This replicates our previous findings [2] which also showed a similar "step-function" of bvFTD and AD memory performance with bvFTD patients showing a lesser memory impairment than AD. Prior studies [19] showed an even milder memory impairment in bvFTD, which might have been due to the inclusion of so called phenocopy cases, who generally do not show memory impairments [2]. Phenocopy cases mimic the behavioral symptoms of bvFTD but do not progress in their disease, show no macroscopic brain atrophy, and perform at control level for most neuropsychological tests. Thus, inclusion of these cases might have led to the misconception that memory is intact in bvFTD.

The imaging results show that orientation performance relates specifically to posterior hippocampus cortex atrophy, whereas memory performance was more related to the anterior hippocampus. The memory deficit in $\mathrm{AD}$ is typically regarded as reflecting medial temporal lobe pathology, with damage even only to the anterior hippocampus causing a substantial deficit in encoding and recall of new information [20]. Our findings corroborate this notion by showing a 

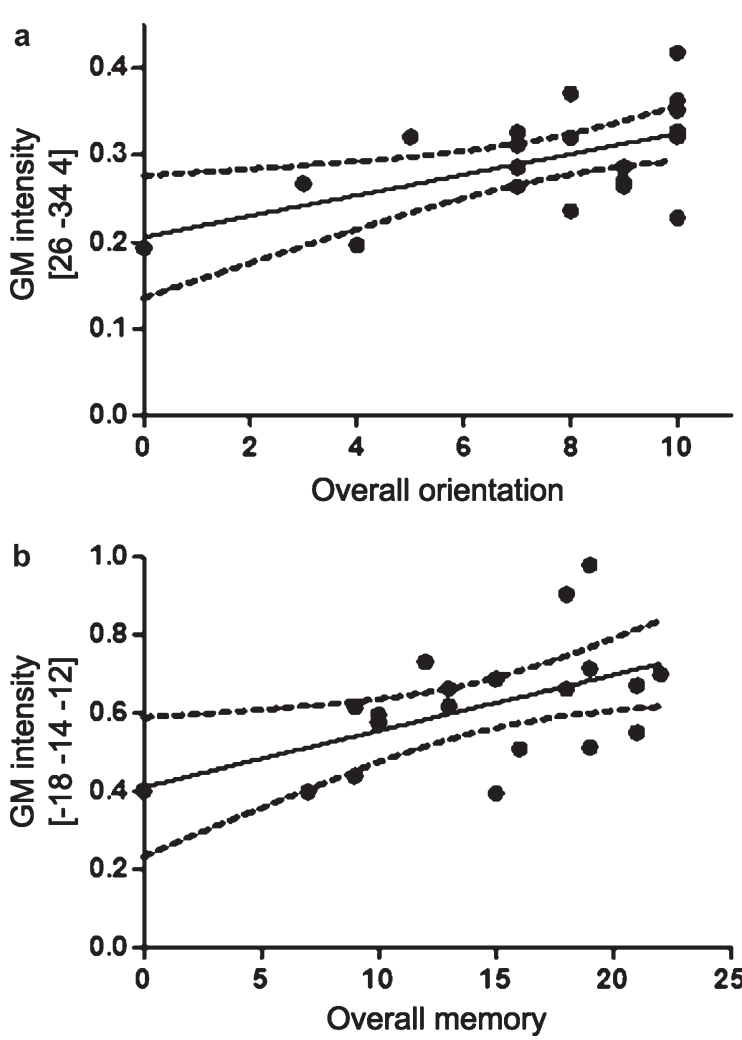

Fig. 3. Correlation between grey matter (GM) intensity and orientation and memory scores. Pearson correlations showing relationship between GM intensity at peak voxels and a) overall orientation and b) overall memory scores. Continuous line indicates a linear coefficient fit between the measures. The dashed line indicates $95 \%$ confidence intervals of the linear coefficient fit.

strong correlation of anterior hippocampus and ACE$\mathrm{R}$ memory performance. Nevertheless, the observed relationship between orientation scores and posterior hippocampus has not been shown before. The overall function of the posterior hippocampus is still debated. Functional neuroimaging studies in healthy participants commonly show activations in the posterior hippocampus/retrosplenial cortex during spatial navigation tasks (e.g., [21]). More importantly, a functional imaging study showed that activity in the posterior hippocampus increased when topographical information was updated or manipulated and also when the topographical information was acquired [22]. These findings are in alignment with our results, as spatial and temporal orientation information was correlated with atrophy in this region. Further, spatial orientation was also associated with anterior hippocampus atrophy, at nearly exact the same location as the memory neural correlates. It is currently not clear why the temporal and spatial orientation information show this different pattern, which clearly needs to be investigated in the future. The imaging results in the bvFTD groups showed only a significant relation between the overall memory score and anterior/middle hippocampal atrophy. Interestingly, only the right hippocampus correlated significantly with memory performance. A stronger right lateralization of atrophy is commonly observed in bvFTD [7] and might therefore have contributed to this finding.

The findings confirm the common clinical observation of severe disorientation in $\mathrm{AD}$, while bvFTD patients can usually provide place and time information with little hesitation. One reason for the dissociation could be the structural integrity of anterior versus posterior hippocampus across both groups. Indeed, AD and bvFTD patients both show hippocampal atrophy, although the magnitude of atrophy in bvFTD varies across patients and can be more focused in anterior hippocampal regions [7], particularly with the tau-negative FTD patients showing more substantial

Table 2

Voxel-based morphometry results showing regions of significant grey matter intensity decrease that covary with memory and orientation performance. All results uncorrected at $p<0.001$; only clusters with at least 50 contiguous voxels included

\begin{tabular}{|c|c|c|c|c|c|c|}
\hline \multirow[t]{2}{*}{ Regions } & \multirow{2}{*}{$\begin{array}{l}\text { Hemisphere } \\
\text { (L/R/B) }\end{array}$} & \multicolumn{3}{|c|}{ MNI Coordinates } & \multirow{2}{*}{$\begin{array}{l}\text { Number of } \\
\text { voxels }\end{array}$} & \multirow{2}{*}{$\begin{array}{c}\text { T score } \\
\text { (peak voxel) }\end{array}$} \\
\hline & & $\mathrm{X}$ & $\mathrm{Y}$ & $\mathrm{Z}$ & & \\
\hline \multicolumn{7}{|c|}{ Orientation overall } \\
\hline Hippocampus & $\mathrm{R}$ & 26 & -34 & 4 & 135 & 2.9 \\
\hline \multicolumn{7}{|l|}{ Memory overall } \\
\hline Hippocampus & $\mathrm{L}$ & -18 & -14 & -12 & 118 & 2.9 \\
\hline \multicolumn{7}{|c|}{ Orientation temporal } \\
\hline Hippocampus & $\mathrm{R}$ & 24 & -34 & 4 & 22 & 2.5 \\
\hline \multicolumn{7}{|l|}{ Orientation spatial } \\
\hline Hippocampus & $\mathrm{R}$ & 22 & -32 & 4 & 149 & 2.9 \\
\hline Hippocampus & $\mathrm{L}$ & -16 & -18 & -14 & 35 & 2.9 \\
\hline
\end{tabular}


i)

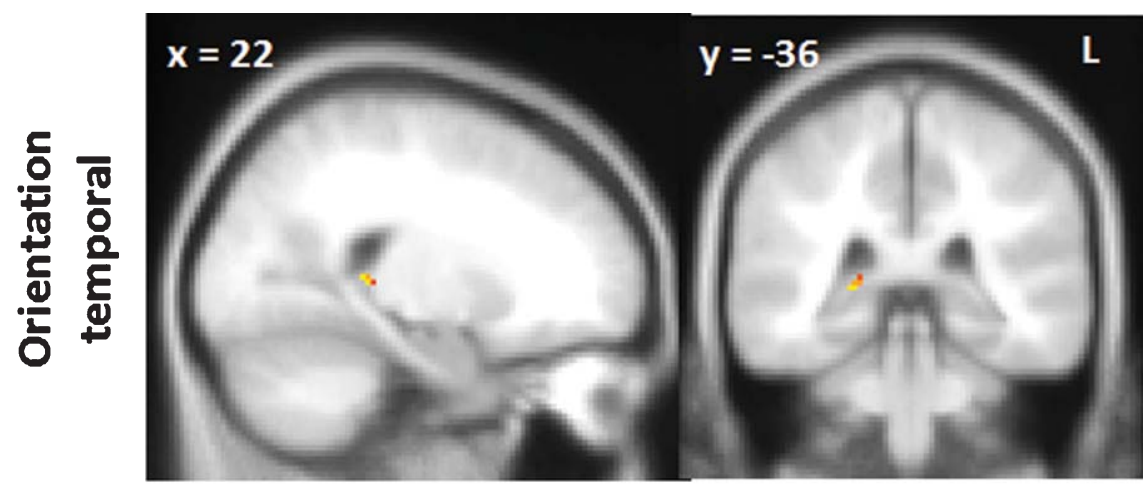

ii)

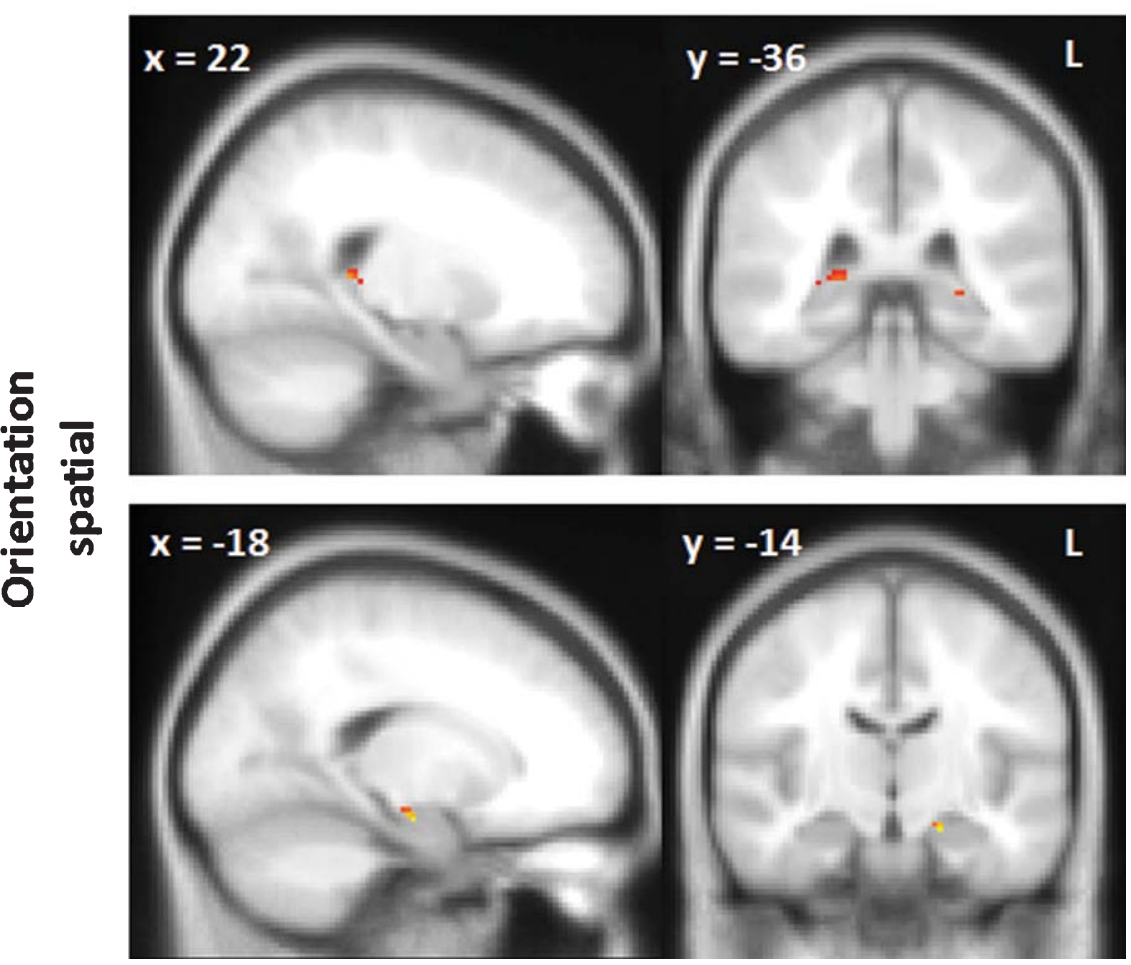

Fig. 4. Brain atrophy related to temporal and spatial orientation. Voxel-based morphometry analyses showing grey matter atrophy covarying with i) temporal and ii) spatial orientation scores in AD only. Clusters are overlaid on the MNI standard brain $(t>2.41)$. Colored voxels show regions that were significant in the analyses for $p<0.0001$ uncorrected and a cluster threshold of 20 contiguous voxels.

hippocampal sclerosis [23]. By contrast, atrophy and hypometabolism of the posterior hippocampus and the adjacent retrosplenial cortex has been shown to already be present in the prodromal stages of $\mathrm{AD}[24$, $25]$, whereas the same brain regions remain intact in bvFTD until the late disease stages [26]. Thus, tests that tap into the function of anterior hippocampus versus posterior hippocampus should dissociate both patient groups. This notion is corroborated by a recent spatial navigation study [27] in mild cognitive impairment (MCI), AD, and semantic dementia (SD) patients, which showed that MCI and AD patients were severely impaired on simple route learning tasks and head orientation tasks. By contrast, the SD patients, showing mostly anterior hippocampal atrophy, performed at controls level on these tasks. Thus, there appears to be a functional dissociation along the axis of the hippocampus which has clinical relevance. Our results dovetail this notion of a functional dissociation by show that a combination of simple orientation and memory measures can distinguish AD and bvFTD in $78 \%$ of cases at presentation. Employment of such simple measures 
might therefore aid diagnosis, in particular when the neuropsychological profile of a patient is not clearly indicative of $\mathrm{AD}$ or bvFTD.

Taken together, our findings show that $\mathrm{AD}$ and bvFTD patients can be discriminated to a large degree on simple orientation and memory measures. More importantly, the orientation and memory measures tap into different neural correlates with the posterior hippocampus involved in orientation and the anterior hippocampus involved in memory recall. This has clear diagnostic implications in that clinicians should take into account orientation measures in combination with memory and other cognitive measures to distinguish $\mathrm{AD}$ from other neurodegenerative conditions.

Unfortunately, we did not have pathological confirmation in the tested cases so we cannot exclude that some of the bvFTD patients might have AD pathology, in particular those who performed very badly at orientation. This is an important point to address in future studies as the NINCDS-ADRDA criteria show only a poor differentiation of AD and FTD [28] and patients with behavioral symptoms may have underlying $\mathrm{AD}$ pathology [29]. Thus Pittsburgh Compound B scanning or cerebrospinal fluid testing of future sample will be important to confirm our results. Still, our memory findings are in keeping with studies using pathological confirmed cases of bvFTD [2,3], supporting therefore the validity of the current findings.

Another shortcoming of the current study is that the imaging data did not survive more conservative multiple comparison corrections procedures (i.e., FWE) and thus is reported uncorrected at $p<0.001$. We tried to therefore reduce the potential of false positive results by introducing a cluster extent threshold of 20 contiguous voxels in our data. The rationale behind such cluster extent thresholding is based on the notion that the probability of a contiguous extent of voxels being all false positive is lower than for any given single voxel. Indeed, Monte Carlo simulations as well as experimental data have shown that cluster thresholding can be an efficient tool to reduce false positives without having significant effects on the statistical power [30]. Still, there is currently no consensus in the literature on the appropriate minimum cluster extent threshold size. This is in particular relevant for studies investigating subcortical structures, such as the hippocampus in our study, as it is not clear whether cluster thresholding disadvantages results in those regions due to their small spatial extent. Not surprisingly, therefore studies exploring hippocampal atrophy have either employed cluster thresholding ([e.g. 31], or not [e.g. 32]. We think our current approach with a cluster extent threshold of
20 contiguous voxels is on the conservative side of studies investigating subcortical atrophy correlates of cognitive dysfunction. Nevertheless, replication of our results by future investigations is very important in particular for the smaller cluster in our results, such as for the orientation subscores. Future investigations should also corroborate our findings in pathologically confirmed cases and also explore more objective, in-depth assessments of orientation.

\section{ACKNOWLEDGMENTS}

This work was supported by an ARC Centre of Excellence grant. MH is supported by an ARC research fellowship (DP110104202).

Authors' disclosures available online (http://www.jalz.com/disclosures/view.php?id=1488).

\section{REFERENCES}

[1] Rascovsky K, Hodges JR, Knopman D, Mendez MF, Kramer JH, Neuhaus J, van Swieten JC, Seelaar H, Dopper EG, Onyike CU, Hillis AE, Josephs KA, Boeve BF, Kertesz A, Seeley WW, Rankin KP, Johnson JK, Gorno-Tempini ML, Rosen H, Prioleau-Latham CE, Lee A, Kipps CM, Lillo P, Piguet O, Rohrer JD, Rossor MN, Warren JD, Fox NC, Galasko D, Salmon DP, Black SE, Mesulam M, Weintraub S, Dickerson BC, Diehl-Schmid J, Pasquier F, Deramecourt V, Lebert F, Pijnenburg Y, Chow TW, Manes F, Grafman J, Cappa SF, Freedman M, Grossman M, Miller BL (2011) Sensitivity of revised diagnostic criteria for the behavioural variant of frontotemporal dementia. Brain 134, 2456-2477.

[2] Hornberger M, Piguet O, Graham AJ, Nestor PJ, Hodges JR (2010) How preserved is episodic memory in behavioral variant frontotemporal dementia? Neurology 74, 472-479.

[3] Graham A, Davies R, Xuereb J, Halliday G, Kril J, Creasey H, Graham K, Hodges J (2005) Pathologically proven frontotemporal dementia presenting with severe amnesia. Brain 128, 597-605.

[4] Rabinovici GD, Seeley WW, Kim EJ, Gorno-Tempini ML, Rascovsky K, Pagliaro TA, Allison SC, Halabi C, Kramer JH, Johnson JK, Weiner MW, Forman MS, Trojanowski JQ, Dearmond SJ, Miller BL, Rosen HJ (2007) Distinct MRI atrophy patterns in autopsy-proven Alzheimer's disease and frontotemporal lobar degeneration. Am J Alzheimers Dis Other Demen 22, 474-488.

[5] van de Pol LA, Hensel A, van der Flier WM, Visser PJ, Pijnenburg YA, Barkhof F, Gertz HJ, Scheltens P (2006) Hippocampal atrophy on MRI in frontotemporal lobar degeneration and Alzheimer's disease. J Neurol Neurosurg Psychiatry 77, 439-442.

[6] Pennington C, Hodges JR, Hornberger M (2011) Neural correlates of episodic memory in behavioral variant frontotemporal dementia. J Alzheimers Dis 24, 261-268.

[7] Whitwell JL, Przybelski SA, Weigand SD, Ivnik RJ, Vemuri P, Gunter JL, Senjem ML, Shiung MM, Boeve BF, Knopman DS, Parisi JE, Dickson DW, Petersen RC, Jack CR Jr, Josephs KA (2009) Distinct anatomical subtypes of the behavioural variant of frontotemporal dementia: A cluster analysis study. Brain 132, 2932-2946. 
[8] Morris JC (1999) Clinical presentation and course of Alzheimer disease. In Alzheimer disease, Terry RD, Katzman R, Bick KL, Sisodia SS, eds. Lippincott Williams \& Wilkins, Philadelphia, pp. 11-24.

[9] Tierney MC, Fisher RH, Lewis AJ, Zorzitto ML, Snow WG, Reid DW, Nieuwstraten P (1988) The NINCDS-ADRDA Work Group criteria for the clinical diagnosis of probable Alzheimer's disease: A clinicopathologic study of 57 cases. Neurology 38, 359-364.

[10] Mioshi E, Dawson K, Mitchell J, Arnold R, Hodges JR (2006) The Addenbrooke's Cognitive Examination Revised (ACE$\mathrm{R})$ : A brief cognitive test battery for dementia screening. Int J Geriatr Psychiatry 21, 1078-1085.

[11] Morris JC (1993) The Clinical Dementia Rating (CDR): Curent version and scoring rules. Neurology 43, 2412-2414.

[12] Ashburner J, Friston KJ (2000) Voxel-based morphometrythe methods. Neuroimage 11, 805-821.

[13] Good CD, Johnsrude IS, Ashburner J, Henson RN, Friston KJ, Frackowiak RS (2001) A voxel-based morphometric study of ageing in 465 normal adult human brains. Neuroimage $\mathbf{1 4}$ 21-36.

[14] Smith SM, Jenkinson M, Woolrich MW, Beckmann CF, Behrens TE, Johansen-Berg H, Bannister PR, De Luca M, Drobnjak I, Flitney DE, Niazy RK, Saunders J, Vickers J, Zhang Y, De Stefano N, Brady JM, Matthews PM (2004) Advances in functional and structural MR image analysis and implementation as FSL. Neuroimage 23(Suppl 1), S208S219.

[15] Zhang Y, Brady M, Smith S (2001) Segmentation of brain MR images through a hidden Markov random field model and the expectation-maximization algorithm. IEEE Trans Med Imaging 20, 45-57.

[16] Rueckert D, Sonoda LI, Hayes C, Hill DL, Leach MO, Hawkes DJ (1999) Nonrigid registration using free-form deformations: Application to breast MR images. IEEE Trans Med Imaging 18, 712-721.

[17] Smith SM, Nichols TE (2009) Threshold-free cluster enhancement: Addressing problems of smoothing, threshold dependence and localisation in cluster inference. Neuroimage 44, 83-98.

[18] Varma AR, Snowden JS, Lloyd JJ, Talbot PR, Mann DMA, Neary D (1999) Evaluation of the NINCDS-ADRDA criteria in the differentiation of Alzheimer's disease and frontotemporal dementia. J Neurol Neurosurg Psychiatry 66, 184-188.

[19] Hutchinson AD, Mathias JL (2007) Neuropsychological deficits in frontotemporal dementia and Alzheimer's disease: A meta-analytic review. J Neurol Neurosurg Psychiatry 78, 917-928.
[20] Sabuncu MR, Desikan RS, Sepulcre J, Yeo BT, Liu H, Schmansky NJ, Reuter M, Weiner MW, Buckner RL, Sperling RA, Fischl B (2011) The dynamics of cortical and hippocampal atrophy in Alzheimer disease. Arch Neurol 68, 1040-1048.

[21] Maguire EA (2001) The retrosplenial contribution to human navigation: A review of lesion and neuroimaging findings. Scand J Psychol 42, 225-238.

[22] Spiers HJ, Maguire EA (2006) Spontaneous mentalizing during an interactive real world task: An fMRI study. $\mathrm{Neu}$ ropsychologia 44, 1674-1682.

[23] Josephs KA, Dickson DW (2007) Hippocampal sclerosis in tau-negative frontotemporal lobar degeneration. Neurobiol Aging 28, 1718-1722.

[24] Pengas G, Hodges JR, Watson P, Nestor PJ (2010) Focal posterior cingulate atrophy in incipient Alzheimer's disease. Neurobiol Aging 31, 25-33.

[25] Nestor PJ, Fryer TD, Ikeda M, Hodges JR (2003) Retrosplenial cortex (BA 29/30) hypometabolism in mild cognitive impairment (prodromal Alzheimer's disease). Eur J Neurosci 18, 2663-2667.

[26] Seeley WW, Crawford R, Rascovsky K, Kramer JH, Weiner M, Miller BL, Gorno-Tempini ML (2008) Frontal paralimbic network atrophy in very mild behavioral variant frontotemporal dementia. Arch Neurol 65, 249-255.

[27] Pengas G, Patterson K, Arnold RJ, Bird CM, Burgess N, Nestor PJ (2010) Lost and found: Bespoke memory testing for Alzheimer's disease and semantic dementia. J Alzheimers Dis 21, 1347-1365.

[28] Varma AR, Snowden JS, Lloyd JJ, Talbot PR, Mann DM, Neary D (1999) Evaluation of the NINCDS-ADRDA criteria in the differentiation of Alzheimer's disease and frontotemporal dementia. J Neurol Neurosurg Psychiatry 66, 184-188.

[29] Alladi S, Xuereb J, Bak T, Nestor P, Knibb J, Patterson K, Hodges JR (2007) Focal cortical presentations of Alzheimer's disease. Brain 130, 2636-2645.

[30] Forman SD, Cohen JD, Fitzgerald M, Eddy WF, Mintun MA, Noll DC (1995) Improved assessment of significant activation in functional magnetic resonance imaging (fMRI): Use of a cluster-size threshold. Magn Reson Med 33, 636-647.

[31] Labate A, Cerasa A, Aguglia U, Mumoli L, Quattrone A, Gambardella A (2010) Voxel-based morphometry of sporadic epileptic patients with mesiotemporal sclerosis. Epilepsia 51, 506-510.

[32] Draganski B, Ashburner J, Hutton C, Kherif F, Frackowiak RS, Helms G, Weiskopf N (2011) Regional specificity of MRI contrast parameter changes in normal ageing revealed by voxel-based quantification (VBQ). Neuroimage 55, 14231434. 